\title{
What Happens to Rotator Cuff Muscles after Rotator Cuff Repair?
}

\author{
Chris Hyunchul Jo $\mathrm{o}^{1,2 \pi}$ \\ ${ }^{1}$ Department of Orthopedic Surgery, SMG-SNU Boramae Medical Center, Seoul National University College of Medicine, ${ }^{2}$ Department of Translational Medicine, \\ Seoul National University College of Medicine, Seoul, Korea
}

Fatty infltration and muscle atrophy have been proposed as important prognostic factors that influence the clinical and structural outcomes of rotator cuff repair, ${ }^{1-3)}$ and both of these are important measures of postoperative functional outcome following rotator cuff repair. ${ }^{4)}$ Thus, accurate assessments of muscle atrophy and fatty infiltration could provide important information with respect to outcomes of rotator cuff repair. Jo et al. ${ }^{1)}$ first reported changes in the appearance of both fatty infiltration and muscle atrophy of rotator cuff muscles on magnetic resonance imaging after rotator cuff repair and suggested that these changes should be considered when assessing changes in rotator cuff muscles by comparing the appearance on magnetic resonance imaging before surgery with that at a certain time after surgery.

A study by Kim et al. "Evaluation of muscular atrophy and fatty infiltration using time-zero magnetic resonance imaging as baseline data, after rotator cuff repair" (Clin Shoulder Elbow. 2019;22(2):70-78) reported changes in fat infiltration and muscle atrophy after rotator cuff repair in 76 patients. Specifically, they compared fat infiltration with Goutallier stage and muscle atrophy with Thomazeau grade and the cross-sectional areas of the supraspinatus between time-zero ( 1 or 2 days after surgery) and 6 months. They found that Goutallier stage and Thomazeau grade did not change significantly, whereas the cross-sectional area of the supraspinatus decreased significantly by $11.41 \%$ $(p<0.01)$ at 6 months. The authors suggested that the decrease was because of medial retraction or strained muscle.

Another study conducted by Jo et al. ${ }^{5}$ and Park et al. ${ }^{6)}$ reported changes in fatty infiltration and muscle atrophy at 1 year after repair. Specifically, they reported that changes in fat infiltration and muscle atrophy after arthroscopic rotator cuff repair differed with respect to baselines and integrity. Generally, both fat infiltration and muscle atrophy measured against the immediate post- operative baseline worsened, while they improved when measured against the preoperative baseline. While muscle atrophy improved in some patients with robust structural integrity after repair, fat infiltration did not reverse in any patients, regardless of structural integrity.

Taken together, these findings indicate that recovery of fatty infiltration and muscle atrophy would rarely occur with current rotator cuff repairs, suggesting further studies and advances in repair strategy are necessary.

\section{References}

1. Jo CH, Shin JS. Changes in appearance of fatty infiltration and muscle atrophy of rotator cuff muscles on magnetic resonance imaging after rotator cuff repair: establishing new time-zero traits. Arthroscopy. 2013;29(3):449-58. doi: 10.1016/j.arthro. 2012.10.006.

2. Thomazeau H, Boukobza E, Morcet N, Chaperon J, Langlais F. Prediction of rotator cuff repair results by magnetic resonance imaging. Clin Orthop Relat Res. 1997;(344):275-83.

3. Liem D, Lichtenberg S, Magosch P, Habermeyer P. Magnetic resonance imaging of arthroscopic supraspinatus tendon repair. J Bone Joint Surg Am. 2007;89(8):1770-6. doi: 10.2106/ JBJS.F.00749.

4. Hata Y, Saitoh S, Murakami N, Kobayashi H, Kaito T, Kato H. Volume changes of supraspinatus and infraspinatus muscles after supraspinatus tendon repair: a magnetic resonance imaging study. J Shoulder Elbow Surg. 2005;14(6):631-5. doi: 10.1016/j.jse.2005.03.012.

5. Jo CH, Park JW, Shin JS. Changes of muscle atrophy according to the immediate postoperative time point in magnetic resonance imaging after arthroscopic rotator cuff repair. Arthroscopy. 2016;32(12):2477-87. doi: 10.1016/j.arthro.2016.04.032.

\section{Correspondence to: Chris Hyunchul Jo}

Department of Orthopedic Surgery, SMG-SNU Boramae Medical Center, Seoul National University College of Medicine, 20 Boramae-ro 5-gil, Dongjak-gu, Seoul 07061, Korea

Tel: +82-2-870-2315, Fax: +82-2-840-2453, E-mail: chrisjo@snu.ac.kr, ORCID: https://orcid.org/0000-0002-6161-5442

Editorial does not need an IRB approval.

Financial support: None. Conflict of interests: None. 
6. Park JW, Jo CH, Shin JS. Changes of fatty infiltration according to the immediate postoperative time point in magnetic resonance imaging after arthroscopic rotator cuff repair. Knee Surg
Sports Traumatol Arthrosc. 2018;26(1):152-60. doi: 10.1007/ s00167-017-4604-9. 